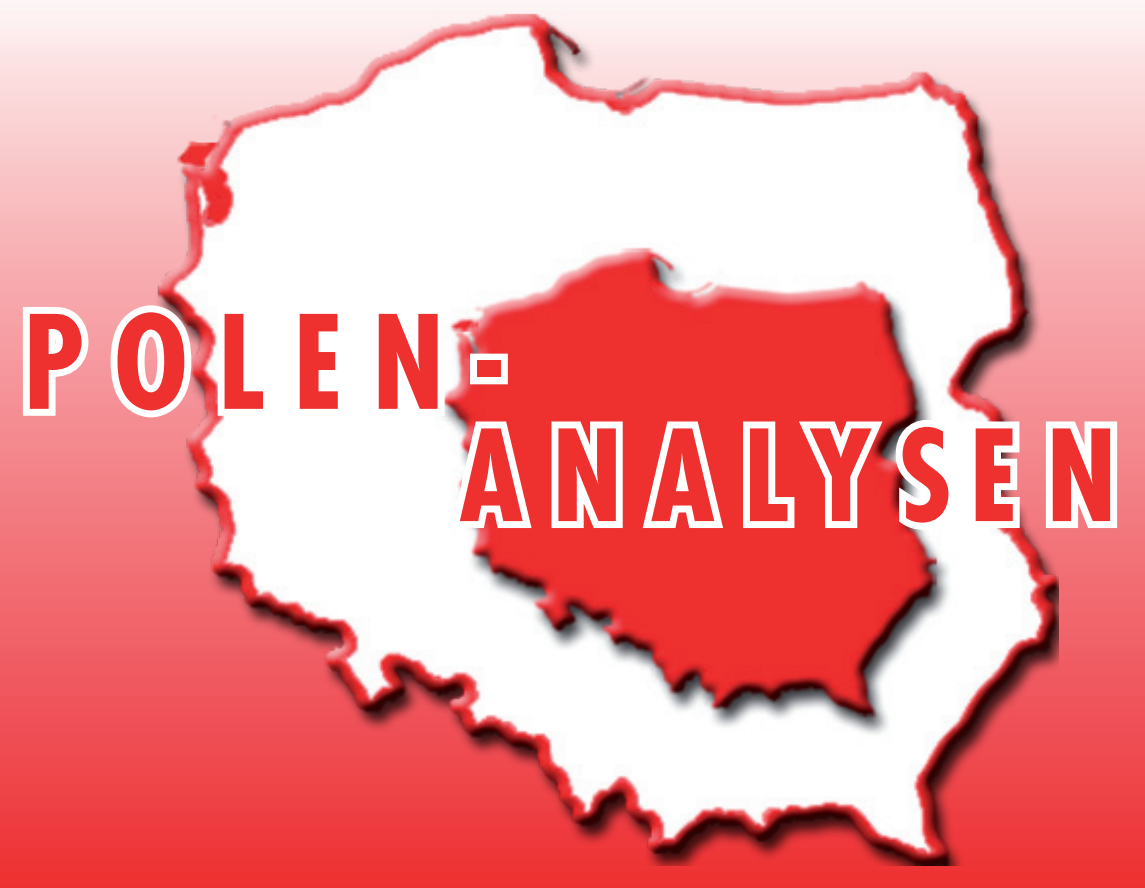

www.laender-analysen.de/polen

\title{
POLNISCHE ENERGIEPOLITIK UND DER UKRAINE-KONFLIKT
}

\section{ANALYSE}

Polnische Energiepolitik und der Ukraine-Konflikt

Michał Olszewski, Krakau

\section{- STATISTIK}

Energieträger, Gaspreis, Energieverbrauch

CHRONIK

6. Mai - 19. Mai 2014

Herausgegeben mit finanzieller Unterstützung der Stiftung für deutsch-polnische Zusammenarbeit

\section{DEUTSCHES} POLEN INSTITUT 


\section{Polnische Energiepolitik und der Ukraine-Konflikt}

Michał Olszewski, Krakau

\section{Zusammenfassung}

Vor dem Hintergrund der enormen Abhängigkeit Polens von russischen Energielieferungen und den Entwicklungen im russisch-ukrainischen Konflikt wirbt Ministerpräsident Donald Tusk aktuell auf europäischer Ebene für eine Energieunion, mit dem Ziel, die allgemein in der EU bestehende Dominanz Russlands einzuschränken. Entgegen der EU-Klimapolitik hält Tusk weiter am Misstrauen gegenüber den erneuerbaren Energien fest, setzt auf die Rehabilitierung der Kohle, was sozialpolitische Konflikte mit der heimischen Bergbaubranche entschärfen würde, und verfolgt langfristig das Ziel der Schiefergasförderung in Polen. Sollten die Vorschläge zur Energieunion konkretere Formen annehmen, werden sie sich mit der EU-Klimapolitik, den divergierenden Energiepolitiken der einzelnen EU-Länder und deren wirtschaftlichen und politischen Beziehungen zu Russland und nicht zuletzt mit der Reaktion Russlands messen müssen.

$I^{2}$

den polnisch-russischen Beziehungen gibt es rituelle

Gesten, die an die Figuren eines klassischen Tanzes erinnern. Das Engagement Polens in Sachen Osteuropa, die Unterstützung der Ukraine oder die Kritik am autoritären Regierungsstil Wladimir Putins rufen immer eine Resonanz hervor: Ins Spiel treten russische Experten, die nachweisen, dass das polnische Fleisch die Gesundheit der russischen Konsumenten gefährden könne, so dass es sich als notwendig erweist, die Lieferungen einzustellen. Im Zusammenhang mit dem Ukraine-Konflikt wuchs die Panik nicht nur vor den polnischen Fleischproduzenten, sondern auch vor den Obstbauern - kam doch aus Moskau das deutliche Signal, dass die Äpfel aus den Plantagen in der Nähe von Warschau einer strengen Kontrolle von Wissenschaftlern unterzogen werden sollten. Wenn dies der Fall wäre, würden wir mit Sicherheit erfahren, dass die polnischen Äpfel übermäßig viele Pestizide aufweisen und den Russen ernste gesundheitliche Folgen drohen, wenn sie sie konsumieren.

Wenn auch die Fleischexporteure das Risiko eines russischen Embargos einkalkuliert haben und die Obstbauern wahrscheinlich auch ohne den östlichen Markt auskommen würden, gibt es doch deutlich ernster zu nehmende Druckmittel. Die Abhängigkeit Polens von den russischen Energierohstoffen ist deutlich höher, als man gemeinhin meint. Das ist nicht nur der berühmt Gashahn, mit dessen Zudrehen man Warschau und einem großen Teil Europas drohen kann. 65 Prozent der nach Polen importierten Kohle kommen aus Russland, sie ist deutlich billiger als der heimische Energieträger. Über 90 Prozent des Erdöls stammen aus russischen Raffinerien. Wenn auch eine eventuelle Unterbrechung der Gaslieferungen Polen nicht von einem Tag auf den anderen lahm legen würde (die Reserven sind ziemlich umfangreich), so könnte eine radikale, aber durchaus vorstellbare Geste in Form einer Reparatur oder Havarie einer von mehreren Raffinerien, die den polnischen Markt bedienen, eine Lähmung hervorrufen. Polen, das muss unterstrichen werden, ist keine Ausnahme auf der europäischen Landkarte: Die Europäische Kommission schätzt, dass, gesetzt den Fall, dass der Import von Energierohstoffen in dem Tempo der vergangenen Jahre weiterwachsen wird, die Europäische Union im Jahr 203040 Prozent der Kohle, 80 Prozent des Erdgases und 90 Prozent des Erdöls einkaufen wird. Im Falle Polens sind die Indikatoren schon jetzt alarmierend, und zwar entsprechend 14, 75 und 100 Prozent. Wenn auch kaum zu erwarten steht, dass Polen Erdöl oder Gasvorkommen hervorzaubern wird, so ist das Importniveau für Kohle doch verwunderlich, wovon später noch die Rede sein wird.

Die Idee der Energieunion von Donald Tusk Es ist daher nicht erstaunlich, dass die Gefahr eines russischukrainischen Krieges eine schnelle Reaktion beim polnischen Ministerpräsidenten Donald Tusk hervorrief, der eine diplomatische Offensive begann und zur Gründung einer Energieunion aufruft. Zunächst fällt es schwer, seine Überlegungen zu kritisieren: Die Energieunion soll die Verhandlungsposition der EU stärken, die zurzeit durch sich eklatant unterscheidende Energieinteressen ihrer Mitgliedsländer gespalten ist. Tusk zufolge ist es jetzt die wichtigste Aufgabe, eine einzige Institution einzuberufen, die im großen Stil Gas für alle $28 \mathrm{EU}-S t a a t e n$ kauft. Wenn man gemeinsam Uran kaufen kann, warum nicht auch Gas? Wenn wir es mit einem Monopolisten zu tun haben, der rücksichtslos seine Auflagen durchdrückt, warum kann man ihm nicht eine einheitliche Abnehmerfront entgegen stellen? Heutzutage sind mindestens zehn EU-Länder von einem einzigen Gaslieferanten abhängig-Gazprom, der über dieHälfte des Verbrauchs deckt. Manche sind vollkommen von dem russischen, staatlich kontrollierten Gasgiganten abhängig, schrieb Tusk in der »Financial Times« im April.

Der polnische Ministerpräsident will außerdem "Solidaritätsmechanismen« aufbauen, mit denen Staa- 
ten unterstützt werden sollen, denen ein Ausschluss von Gaslieferungen droht. Außerdem schlägt er die Finanzierung der wichtigsten Gasinvestitionen durch die EU für solche Länder vor, die am stärksten von Gazprom abhängig sind. Ein weiteres Fundament für die EU wäre auch die Ausnutzung von fossilen Brennstoffvorkommen der EU, darunter Kohle und Schiefergas.

Solcherart Aktivitäten werden deutlich durch die aktuelle Situation begünstigt. Die Aktionen Moskaus zeigen klar, dass es nicht als glaubwürdiger Partner behandelt werden kann. Auch die Zahlen vermögen nicht darüber hinweg zu täuschen, wer die Karten auf dem EU-Energiemarkt verteilt: Im Jahr 2012 betrug der Anteil des russischen Erdgases und Erdöls am Import der EU 30 bzw. 35 Prozent. Daraus erklärt sich sicherlich die Reaktion der Bundeskanzlerin Angela Merkel, die nach anfänglicher Zurückhaltung gegenüber der Idee, den Energiemarkt der EU zu integrieren, die Einzelheiten diskutieren will. Ermutigend wirkt auch die Reaktion des französischen Staatspräsidenten François Hollande, der die Energieunion als "schönes Projekt« beurteilte. Tusk sprach auch mit dem Präsidenten des Europäischen Rates, Herman Van Rompuy, dem Energiekommissar Günther Oettinger und den Ministerpräsidenten von Italien, Spanien und Portugal, Matteo Renzi, Mariano Rajoy und Pedro Passos Coelho.

Soviel zum Allgemeinen und den nicht allzu weit gehenden Absichtserklärungen. Betrachten wir nun die Einzelheiten des Unternehmens.

\section{Misstrauen gegenüber erneuerbaren Energien}

Vor allem fällt in Donald Tusks Konzept ein vielsagender Mangel ins Auge. In gewisser Weise existiert die Energieunion schon seit langem auf dem europäischen Kontinent, und zwar unter dem Namen Klimapolitik. Diese ruht auf einigen Säulen, wozu auch die Notwendigkeit der Abkehr von fossilen Brennstoffen gehört sowie die Vergrößerung der Rolle der erneuerbaren Energien, die Tusk in seinem Projekt mit keinem Wort erwähnt. Das sollte nicht weiter erstaunen, denn das Misstrauen Tusks gegenüber den erneuerbaren Energien ist schon sprichwörtlich und symbolisch für die ganze polnische politische Klasse, die überzeugt ist, dass die grüne Energie ein pfiffig maskierter Betrug ist, der dazu dient, dem Steuerzahler Geld aus der Tasche zu ziehen, und weder die Energieprobleme Polens und noch weniger des europäischen Kontinents löst. Aus polnischer Sicht kann das Experiment unter dem Begriff Energiewende nicht mit einem Erfolg enden; es herrscht die Überzeugung vor, dass dies eine kostspieliger Irrtum sei, für den die Deutschen teuer bezahlen werden.

Ministerpräsident Tusk hat sein Misstrauen gegenüber den erneuerbaren Energien vielmals zum Ausdruck gebracht. Zwar unterstreicht er, dass Polen die aus dem Klimapakt resultierenden Verpflichtungen erfüllen soll, aber nur in Minimalform. Die Zukunft Polens bleibt weiterhin die Kohle als günstigste und heimische Energiequelle, hinzu kommen Atomenergie und Gas - so lässt sich in größter Kürze die Energiepolitik der Regierung zusammenfassen. Allein in den Jahren 2011 - 2012 lehnten es die Operatoren der Verteilersysteme ab, erneuerbare Energiequellen von einer Gesamtkapazität von 5,6 Gigawatt ins Netz einzuspeisen. Das ist so viel, wie die Kapazität von neun großen Kraftwerken.

Obwohl sich die polnische Wirtschaft zunehmend auf importierte Kohle stützt und die Kosten für Kohlenergie sehr hoch sind, wird erneuerbare Energie in Polen immer noch mit Misstrauen behandelt, wie ein technologischer Gedanke, den die Deutschen zu verkaufen versuchen. Daher werden auch weiterhin nur vorgetäuschte Aktivitäten unternommen, die zwar die Empfehlungen der EU erfüllen, gleichzeitig aber nicht die Strukturen des von großen Konzernen dominierten Energiemarktes berühren sollen. Gemäß dem Gesetzesprojekt über die Erneuerbaren Energien wurde der Prosument (=Verbraucher und Produzent) zwar von der Pflicht, eine wirtschaftliche Tätigkeit auszuüben, entbunden, aber gleichzeitig ist der Preis, für den er die von ihm produzierte Energie verkaufen und ins Netz einspeisen kann, so niedrig, dass dies von einer solchen Tätigkeit abhält. Außerdem will der Staat auch die Unterstützung von pseudoökologischen Mitverbrennungen (Kohle wird gleichzeitig mit Biomasse verbrannt) aufrechterhalten, nur dass diese noch schwieriger zu untersuchen sein werden als bisher.

Das Schicksal des Gesetzes über die Erneuerbaren Energien ist ein ideales Symbol für das Misstrauen polnischer Politiker gegenüber den neuen Energiequellen: Die Arbeiten am Gesetz ziehen sich schon seit einigen Jahren, weitere Projekte haben sich in den Vereinbarungen zwischen den Ressorts festgefahren, die Juristen, die für die von der Energiebranche erwartete Form des Dokumentes verantwortlich sind, wechseln. Diese Hängepartie ist für die Kohlebranche und die staatlichen Energiegesellschaften von Vorteil. Die Regierung wehrt sich mit Händen und Füßen vor Lösungen, die den Produzenten der dezentralen Stromerzeugung mehr Freiheiten gewähren. Das Ergebnis? Die Entwicklung der Branche in Polen wird aufgehalten, es mangelt an Entscheidungen, die die Entwicklung von Offshore-Windparks ermöglichen, Misstrauen der Prosumenten, fehlende rechtliche Bestimmungen.

Aus dieser Perspektive sehen Tusks Vorschläge wie der Versuch eines diplomatischen Angriffs aus, der die tatsächlichen Mängel der Armee verdecken soll. Es sei daran erinnert, dass zwischen Polen und der EU seit 
Jahren ein erbitterter diplomatischer Kampf stattfindet. Die polnische Regierung hält nicht nur die Klimapolitik auf, sondern verzögert auch konsequent die Umsetzung einzelner Direktiven des Energie-KlimaPaketes. Die EU hat ihrerseits bereits gelernt, das polnische Veto in Sachen Reduktion von Treibhausgasen zu umgehen. Ende April schrieb im Magazin "Forbes" Marcin Stoczkiewicz, der Vorsitzende der Organisation ClientEarth Polska, der Juristen angehören, die sich mit Umweltrecht beschäftigen: » Ein gutes Beispiel für diese Taktik war das Spiel um die Rücknahme eines Teils der Emissionsberechtigungen, mit dem Ziel, ihren zu niedrigen Preis zu heben. Die polnische Regierung tat, was sie konnte, um dieses nicht zuzulassen. Die Kommission leitete den Entscheidungsprozess allerdings derart, dass eine einstimmige Entscheidung umgangen wurde, wohl wissend, dass die polnischen Verhandlungspartner keine Chance haben, Unterstützer zu finden.«

Die Idee einer Energieunion sieht also wie die Flucht nach vorn aus. Aus der Defensive tritt Donald Tusk in eine aktive Rolle, weist die Richtung für die Entwicklung und zeigt die Pläne für die Zukunft. Fatale Fehler und Außerachtlassungen werden von neuen Vorschlägen überdeckt, die letztlich zu einer Revision der EU-Klimapolitik führen sollen, zumal im Hintergrund der Vorschläge von Tusk der Kampf um die Klimaziele der EU bis 2030 tobt. Polen stellt sich gemeinsam mit den anderen Ländern der Visegrád-Gruppe (Slowakei, Tschechien und Ungarn), Rumänien und Bulgarien gegen die Verpflichtung einer bis zu 40-prozentigen Reduktion der Emissionen bis zum Jahr 2030, worum sich die Länder der informellen Gruppe »Green Growth«, darunter Deutschland, Italien, Frankreich und Großbritannien, bemühen.

\section{Unterhaltung der kostspieligen Bergbaubranche}

Ein anderer Faden, den man beachten muss, ist der Versuch, Kohle als Energiequelle zu rehabilitieren. Der polnische Kontext ist hier besonderer Art. Auf der einen Seite ist die polnische Regierung eine Geisel der Bergbaubranche. Der Steinkohlebergbau beschäftigt zurzeit zirka 100.000 Personen und fährt trotz der seit Jahren dauernden Restrukturierungen weiter Verluste ein. Auf den Halden türmen sich Millionen Tonnen nicht verkaufter Kohle - lieber versorgen sich die Abnehmer mit sibirischer Kohle. Das polnische Rentensystem wird von einer Reihe von Privilegien für die Bergleute belastet. Im vergangenen Vierteljahrhundert haben der Staat und die Steuerzahler den Bergbau auf sehr unterschiedliche Weise unterstützt. Die Gelder flossen nicht nur in die notwendige Restrukturierung (Anfang der 1990er Jahren beschäftigten die Bergwerke zirka 400.000 Personen), Schließung unrentabler Betriebe oder in nicht gerade kleine Abfindungen.
Maciej Bukowski und Aleksander Śniegocki, Autoren der unlängst veröffentlichten »Analyse der wirtschaftlichen Unterstützung für die Kohleenergie sowie den Bergbau in Polen" (Analiza wsparcia gospodarczego dla energetyki weglowej oraz górnictwa $w$ Polsce), weisen darauf hin, dass die staatlichen Institutionen die Rückzahlungen enormer Verpflichtungen eingestellt oder ausgesetzt haben, als da wären Steuern, zustehende Beiträge für die Sozialversicherungsanstalt (ZUS), Abgaben für Bergschäden. Ökonomen haben ausgerechnet, dass die Zuwendungen für den Bergbau in den Jahren 1990 bis 2003 insgesamt 2 Prozent des Bruttoinlandsprodukts betrugen, $d$. h. fünfmal mehr als die damaligen Ausgaben für Wissenschaft und Entwicklungen. Natürlich waren diese beiden Momente die radikale Konsequenz einer ausgeprägten Sozialpolitik, die den Kohlesektor als einen der Bereiche behandelt, der unter dem besonderen Schutz des Staates steht. Der Streit, ob dieser sinnvoll war, hat heute keine größere Bedeutung mehr, sein Höhepunkt liegt hinter uns. Dennoch ist der polnische Staat nicht in der Lage, das Ausnahmesystem der Renten und Pensionen in der Bergbaubranche zu verändern. Nach offiziellen Schätzungen erhöht jeder von einem Bergmann bei der Sozialversicherungsanstalt eingezahlte Zloty die Verpflichtungen ihm gegenüber auf 1,5 bis 1,8 Zloty (abhängig von der ausgeführten Tätigkeit), anstatt 1 Zloty anzulegen, wie im allgemeinen System. Wenn aber die Berechnungen des Warschauer Instituts für Ökonomische Studien (WISE) korrekt sind, dann sind diese Daten deutlich zu niedrig veranschlagt. Warschauer Ökonomen schätzen, dass die im Jahr 2013 vom Staat beglichene Differenz nicht 0,8 Zloty, sondern 1,52 Zloty betrug. Beeinflusst wird dies von der Dauer des Rentenbezugs (die im Falle der Bergleute statistisch gesehen neun Jahre länger ist als im allgemeinen System) sowie vom niedrigen Rentenalter (48 Jahre). Der Anstieg der Gehälter und die längere Lebenszeit verursachen daher, dass das Problem wächst, anstatt sich zu verringern. Das Ausmaß des Ganzen zeigen folgende Schätzungen: In den Jahren 1990 bis 2012 betrugen die Zuwendungen und Subventionen für den Bergbausektor insgesamt $136 \mathrm{Mrd}$. Zloty. Hinzugefügt werden muss die Unterstützung für die Kohleenergie, die vor allem mit den Energierechnungen bezahlt wird. In den Jahren 2005 bis 2012 war dies eine Summe von zirka 42 Mrd. Zloty.

Jedwedem Versuch, diese Struktur zu verändern, droht ein Ausbruch sozialer Unruhen in Oberschlesien. Aus dieser Perspektive erscheinen die Vorschläge von Tusk, der - in seinem Konzept unpräzisiert - Erleichterungen für die Kohlebranche will, verständlich. Da ist aber auch die Kehrseite der Medaille. Nach dem Bericht der Obersten Kontrollkammer (NIK) reichen die polnischen Steinkohlevorkommen bis ungefähr zum Jahr 
2035. Der betriebene Raubbau und die billigsten Abbaumethoden haben, so die Kontrolleure, zu einem unumkehrbaren Verlust eines Teils der Flöze geführt. Dies ist eine sehr starke These, die von den Chefs der Kohlegesellschaften kritisiert wird. Aber auch wenn die Oberste Kontrollkammer übervorsichtig sein sollte, sollten die Warnungen wahrgenommen werden. Polen ist aktuell ein Nettoimporteur von Kohle. Diese Abhängigkeit wird wachsen. Dennoch sind weitere Kohlekraftwerke in Polen geplant. Die Kanzlei des Ministerpräsidenten verfasste im vergangenen Jahr einen Bericht, der zeigte, wie der polnische Energiemix bis zum Jahr 2060 aussehen wird. Es ist ein seltsamer Zufall, dass nach vielen Analysen genau das dabei heraus kam, was mit der konsequent umgesetzten Vision Tusks übereinstimmt: Kohle war, ist und wird am günstigsten sein, auf sie bauen wir unsere Energiestabilität. Die erste Version des Berichtes enthielt noch kardinale Fehler - nicht einmal erwähnt wurden die Energieproduktion aus dezentraler Stromerzeugung oder die externen Kosten der Kohlewirtschaft -, so dass man schließen kann, dass den Autoren des Berichts sehr am Aufbau einer alternativlosen Vision gelegen hat. Was nicht verwunderlich ist - je schwärzer das Bild wird, desto leichter kann man die Gesellschaft vom Bau weiterer Kohlekraftwerke überzeugen und die Europäische Union, Polen von den Regeln der gemeinsamen Klimapolitik auszuschließen.

Der Konflikt in der Ukraine kam Donald Tusk in gewisser Weise bei der Suche nach einem Ausweg aus dem Energie-Sozial-Clinch zu Hilfe, denn der Vorschlag, Kohle wieder in Gnaden aufzunehmen, betrifft verschiedene Bereiche. Erstens wird den Bergleuten gezeigt, dass sich die Regierung für ihre Probleme interessiert. Zweitens eröffnet er die Diskussion darüber, was die polnischen Eliten eh nie akzeptiert haben: Die Gefahr eines Krieges und die Einstellung der Rohstofflieferungen aus dem Osten gibt die Möglichkeit, das Fundament der EU-Klimapolitik in Frage zu stellen. Denn was könnte sich sonst unter der allgemein gehaltenen Formulierung einer "größeren Nutzung der Kohle" verbergen? Überträgt man dies in eine konkretere Sprache, zeigt sich hier der Wunsch nach Zuwendungen, nach Außerkraftsetzung und vielleicht auch nach dem Ausschalten des Auktionssystems. Trotz der Bemühungen der EU-Vertreter wächst der globale Ausstoß von Kohlendioxid. Ein großer Teil der größten Wirtschaften der Welt kümmert sich nicht um das Kyoto-Protokoll und die Notwendigkeit, die Emissionen zu reduzieren. Donald Tusk ist ein Sprecher dieses Teil der öffentlichen Meinung (die nicht nur in Polen vertreten ist), die findet, dass die Umweltpolitik der EU zu restriktiv ist und allerschnellstens gelockert werden sollte. Die Perspektive eines Krieges gibt dafür einen hervorragenden
Vorwand. Wenn jemand ambitioniertere Reduktionsziele haben und weniger Kohlendioxid ausstoßen will, werden wir ihn nicht dabei aufhalten, sagte der polnische Ministerpräsident unlängst. Diese Worte passen sehr gut zum Konzept der Energieunion aus Warschau.

\section{Hoffnung Schiefergas?}

Etwas anders stellt sich die Förderung von Schiefergas dar, von der Regierungschef Tusk spricht. Kann man im Fall der Kohle vom Versuch einer Rehabilitierung sprechen, so bezieht sich das Engagement für Schiefergas auf eine nicht näher bestimmte Zukunft. In der Tat hat die Europäische Kommission nach der verbissenen Schlacht, an der Polen deutlichen Anteil hatte, keine restriktiven Regeln für den Abbau von Schiefergas eingeführt. Andererseits ist von der Euphorie, die vor einigen Jahren geherrscht hatte, als die Polen glaubten, dass in Kürze an der Weichsel die Gasförderung in großem Stil beginnt und die Lieferungen aus Russland entbehrlich werden, keine Spur geblieben. Es zeigte sich, dass die Vorkommen nicht so groß sind, wie Wissenschaftler anfänglich angenommen hatten, und ihre Tiefe wird den Förderprozess um ein Mehrfaches teurer machen als in den USA. Anders gesagt: In den nächsten Jahren wird Schiefergas wahrscheinlich weder die Energieprobleme Polens noch eines anderen Landes in Europa lösen, was natürlich nicht von der Notwendigkeit entbindet, den rechtlichen und sozialen Rahmen für die eventuelle Förderung festzulegen.

Die Vision eines Schiefergas-Eldorado sollte allerdings auch nicht die gewichtigen Schwierigkeiten verdecken, die sich unabänderlich mit dieser Energiequelle verbinden. Die Landwirte, die seit über einem Jahr in Żurawlów (Ostpolen) protestieren, sind ein deutliches Beispiel: Die Förderung, das geben sogar die größten Fürsprecher zu, zerstört die Landschaft und erschwert die landwirtschaftliche Produktion. Polen, dessen Besiedlung dreimal höher ist als in den USA, wird also eher unter diesem Aspekt ein Konfliktfeld, und zwar zwischen denen, die bohren, und denen, die säen wollen. Das erste europäische Land, in dem Schiefergas ins Verteilernetz fließen wird, ist Großbritannien. Allerdings wird auch dort die industrielle Förderung frühestens in einem Jahr beginnen.

\section{Die Energieunion und die Herausforderungen der nationalen Politiken} Eine gesonderte Frage, die die Politiker bisher nicht berührt haben, sind die sehr unbequemen Konsequenzen einer tatsächlichen Energieunion. Vorerst hat die Ethik gegenüber der Pragmatik den Vorrang, was nicht verwunderlich ist, umso weniger, als diese Geste nicht viel kostet. Geschmeidige Erklärungen der europäischen politischen Führungskräfte ändern nichts an der Tatsache, dass jedes EU-Land zurzeit seine eigene Energie- 
politik betreibt, was u. a. durch den Lissaboner Vertrag garantiert wird. Mehr noch, einige EU-Länder führen mit Russland sehr unterschiedliche Geschäfte, und es ist schwer vorstellbar, dass sie im Namen der Interessen Polens darauf verzichten werden. Frankreich, das sich die Idee der Energieunion zu eigen machte, ist mit Moskau in umfangreichen verteidigungs- und energiepolitischen Geschäften eng verbunden. Ungarn hat sich im Verlauf des Konfliktes auf der Krim entschieden für Moskau ausgesprochen und die Gaslieferungen in die Ukraine auf ein Minimum beschränkt, um den östlichen Partner nicht zu ärgern. Eine ähnliche Geste kam aus der Slowakei. Gegen die Einfrierung des South Stream Projektes sprachen sich deutlich die Länder des südlichen Gürtels der EU aus, von Bulgarien bis Italien. Auch ist schwer vorstellbar, dass Angela Merkel nicht den Ostausschuss der Deutschen Wirtschaft im Blick haben wird, der 180 Firmen versammelt, die in Russland oder Kasachstan investieren. In der Zeit, als Russland die Krim einnahm, haben sich deren Vertreter entschieden den Versuchen entgegen gestellt, Druck auf den östlichen Partner auszuüben. Es fällt also schwer, zu erwarten, dass eine eventuelle Energieunion in Moskau anders wahrgenommen würde, als ein Schlag in die eigenen Lebenskräfte. Falls es also zu fortgeschrittenen Gesprächen zwischen den einzelnen Ländern der EU kommen sollte, muss damit gerechnet werden, dass aus Russland eindeutige Signale der Missbilligung kommen werden. Dabei geht es dann nicht mehr nur um polnische Äpfel oder Schweinefleisch, sondern um französische Kriegsschiffe, das Recht, in der Arktis Rohstoffe abzubauen, und um Geschäfte in Kasachstan. Es kann auch sein, dass Russland, zumindest im Energiebereich, beginnen wird, eine aktivere Preispolitik zu betreiben, Veränderungen in der Marge für bestimmte Länder vornehmen wird und auf diese Weise das Interesse an einem gemeinsamen Energiemarkt in der EU verwässern wird. Der Umsetzung des Planes kann auch etwas im Wege stehen, was Gregorz Onichimowski, Vorsitzender von WSE InfoEngine S.A. (einem Dienstleister im Bereich Energiehandel und -beratung), treffend als "Philosophie der Energieeinkäufe« bezeichnete. Im Internetportal »defence. 24 « verglich Onichimowski zwei völlig verschiedene Bezahlungssysteme, und zwar von Polen und von Deutschland. In Polen bezahlen die industriellen Verbraucher die höchsten Sätze für Energie. In Deutschland, wo davon ausgegangen wird, dass es besser sei, dass der Durchschnittsbürger Arbeit hat, zahlt die Industrie sogar um den Preis letztlich höherer Kosten den niedrigsten Satz. Solche Unterschiede können die Suche nach einem gemeinsamen Nenner für die 28 EU-Mitgliedsländer erschweren.

Die Idee eines gemeinsamen Gasmarktes liegt zurzeit noch im Nebel. Vermutlich geht es Ministerpräsi- dent Donald Tusk nicht darum, dass einzelne Länder für gemeinsame Einkäufe zusammenlegen und anschlieBend den blauen Brennstoff aufteilen, sondern es geht um den Bau von Knotenpunkten, an denen der Großhandelsumsatz der Ware Gas stattfindet, um Verbindungen über Staatsgrenzen hinweg und um den Bau neuer Terminals.

Dies klingt wenig kontrovers, es kann sich allerdings erweisen, dass, wenn die Politik von der Ebene bequemer Allgemeinplätze ins Konkrete hinabsteigt, sich die Diskrepanzen als zu breitgefächert darstellen und Polen in seinen Bemühungen allein dasteht. Das Risiko einer Niederlage der Energieunion ist also groß. Was wird passieren, wenn ihr Ideengeber nicht in der Lage ist, sein Konzept durchzusetzen? Polen versucht, auf eine solche Situation vorbereitet zu sein. Bei allen Vorbehalten gegenüber der polnischen Energiepolitik, die häufig unlogisch und misslungen ist, muss man eines zugeben: Die polnischen Politiker haben ihre Lehre aus der Gaskrise im Januar 2009 gezogen, als der Streit zwischen Russland und der Ukraine dazu geführt hatte, dass der Gashahn zugedreht worden war, was eine Krise in Mitteleuropa nach sich gezogen hatte. Seitdem ist die polnische Energiewirtschaft deutlich besser auf solcherart Krisensituationen vorbereitet. Es wurden Magazine gebaut, deren gesamtes Fassungsvermögen die kontinuierliche Lieferung für Industrie und Bevölkerung für zirka zwei Monate garantiert. In diesem Jahr wird der Gashafen in Swinemünde (Świnoujście) eröffnet, so dass Polen Flüssiggaslieferungen aus Katar in Empfang nehmen kann. Interessanterweise ist dies der einzige Bereich der polnischen Energiepolitik, der nicht Gegenstand des Streites mit der EU ist.

Dies ändert jedoch nichts an der Tatsache, dass man, wenn man die Angst vor dem Mittel des zugedrehten Gashahns ernst nimmt, mit dem der große Nachbar ständig droht, eine unbequeme Frage stellen muss, die in den Gesprächen zwischen Tusk und den führenden Köpfen einzelner EU-Länder nicht fiel. Was hat Polen in den letzten Jahren getan, um seine Abhängigkeit von dem aus Russland importierten Brennstoff zu verringern? Die Antwort lautet: Viel, aber deutlich weniger als es hätte tun können. Die Blockade der Entwicklung erneuerbarer Energiequellen und der energetischen Effektivität unterstützt quasi aus eigenem Antrieb die Abhängigkeit von Russland. Die aktuellen Ereignisse in der Ukraine haben in dieser Hinsicht nichts verändert. Wind, Biomasse und Sonne sind immer noch ebenso verdächtig wie der blaue Brennstoff, der aus den Gaspipelines aus dem Osten kommt.

Übersetzung aus dem Polnischen: Silke Plate Information über den Autor finden Sie auf der nächsten Seite. 
Über den Autor

Michał Olszewski, Publizist und Schriftsteller, hat sich im Bereich Journalistik auf ökologische Themen spezialisiert und schreibt in meinungsführenden polnischen Zeitungen u. a. über die Kohlewirtschaft und die Klimapolitik.

\section{STATISTIK}

\section{Energieträger, Gaspreis, Energieverbrauch}

Tabelle 1: Angenommene Förderung von Energierohstoffen mit Schlüsselbedeutung in Polen bis 2050

\begin{tabular}{|c|c|c|c|}
\hline & 2010 & 2030 & 2050 \\
\hline Steinkohle & 76 Mio. $t$ & 47 Mio. t & 28 Mio. $\mathrm{t}$ \\
\hline $\begin{array}{l}\text { Braunkohle (ohne neuen } \\
\text { Tagebau) }\end{array}$ & 61 Mio. $t$ & 46 Mio. $t$ & 0 \\
\hline $\begin{array}{l}\text { Braunkohle } \\
\text { (Aufrechterhaltung des } \\
\text { Abbaus) }\end{array}$ & 61 Mio. $\mathrm{t}$ & 60 Mio. $\mathrm{t}$ & 60 Mio. $\mathrm{t}$ \\
\hline $\begin{array}{l}\text { Braunkohle (Steigerung des } \\
\text { Abbaus) }\end{array}$ & 61 Mio. $\mathrm{t}$ & 95 Mio. $t$ & 110 Mio. $t$ \\
\hline Erdgas & $5 \mathrm{Mrd} . \mathrm{m}^{3}$ & 8 Mrd. $\mathrm{m}^{3}$ & 9 Mrd. $\mathrm{m}^{3}$ \\
\hline Erdöl & 0,8 Mio. $\mathrm{t}$ & 0,2 Mio. $\mathrm{t}$ & 0,1 Mio. $\mathrm{t}$ \\
\hline
\end{tabular}

Quelle: Bukowski, Maciej (Hg.): 2050.pl. Podróż do niskoemisyjnej przyszłości [Die Reise in eine Niedrig-Emissions-Zukunft]. Warszawa 2013, <http://np2050.pl/files/pliki/NP_2050__CALOSC_internet_2.pdf> (abgerufen am 15.05.2014).

Grafik 1: Der Gaspreis für einzelne europäische Länder, verkauft von Gazprom (Dollar pro $1000 \mathrm{~m}^{3}$ )

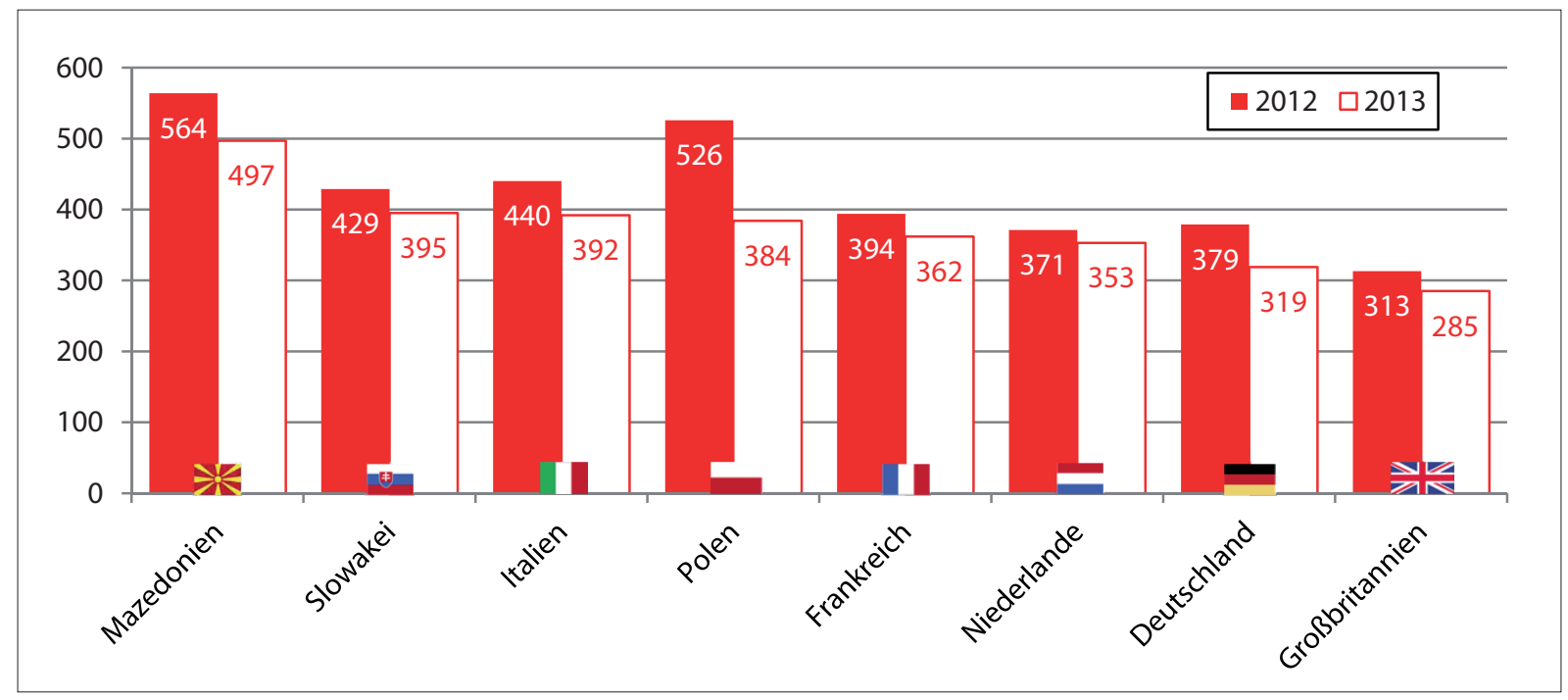

Quelle: Gazeta Wyborcza/Izwiestia/Gazprom: <http://wyborcza.biz/biznes/1,100896,13348060,Czy_Polska_przestanie_placic_ najdrozej_w_UE_za_gaz.html> (abgerufen am 15.05.2014). 
Grafik 2: Die Struktur des Verbrauchs von Primärenergieträgern in Polen (Anteil an der produzierten Energie insgesamt)

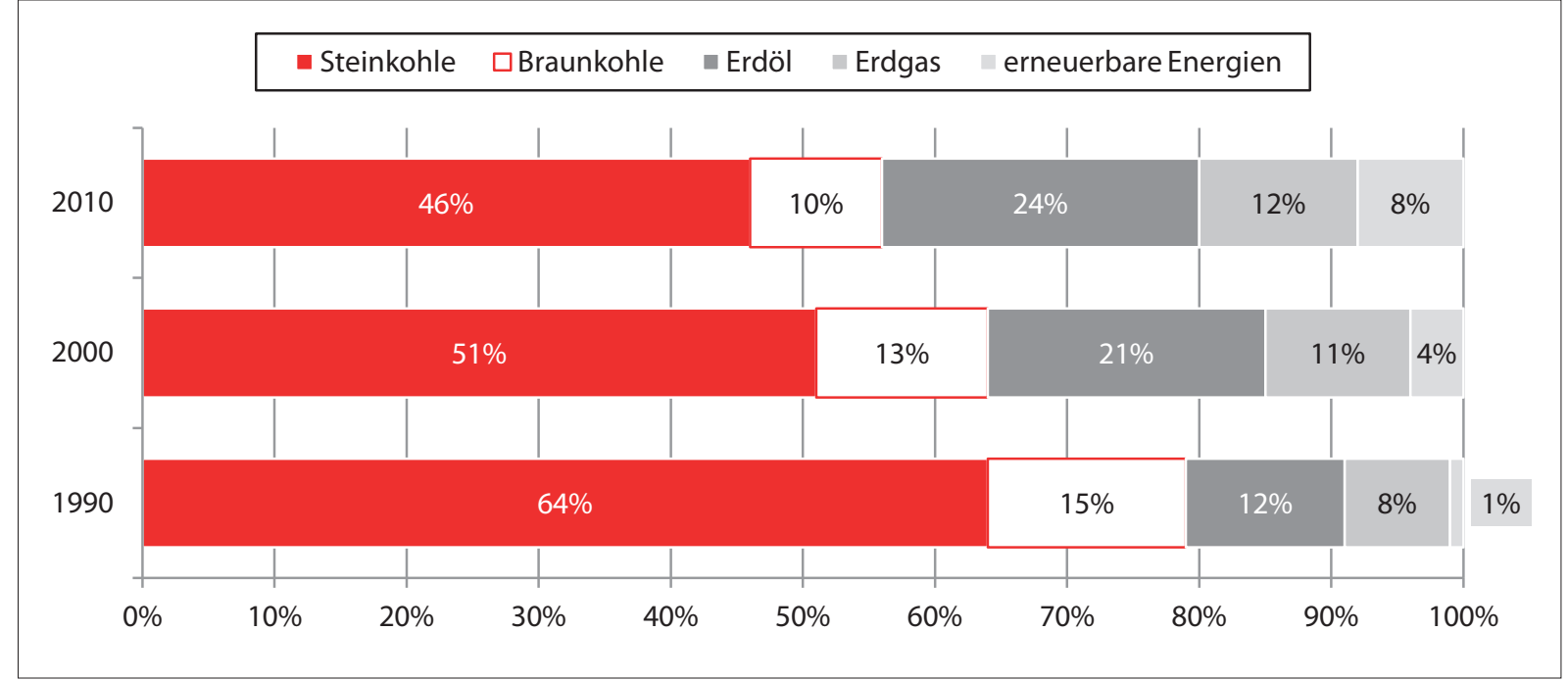

Quelle: Wilczyński, Michat: Zmierzch węgla kamiennego w Polsce [Der Niedergang der Steinkohle in Polen]. Warszawa: Instytut na Rzecz Ekorozwoju 2013.

Grafik 3: Die Struktur des Verbrauchs von Primärenergieträgern in Deutschland (Anteil an der produzierten Energie insgesamt)

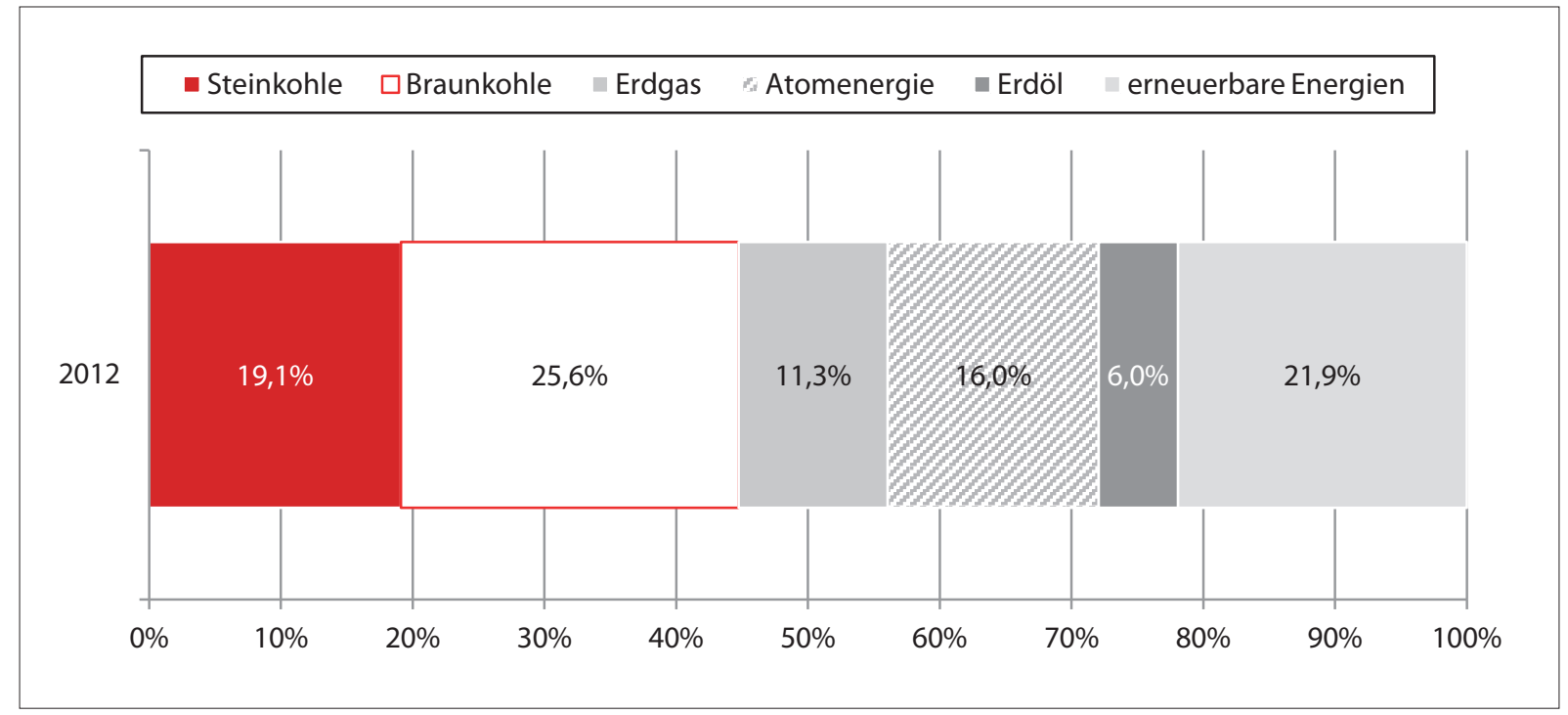

Quelle: Bundesverband der Energie- und Wasserwirtschaft, AG Energiebilanzen, 12/2012 <https://www.hcn.org/blogs/goat/images-2/ GermanEnergyMix.jpg> (abgerufen am 15.05.2014). 


\section{Grafik 4: Die Energieintensität der Wirtschaft in Polen und Deutschland}

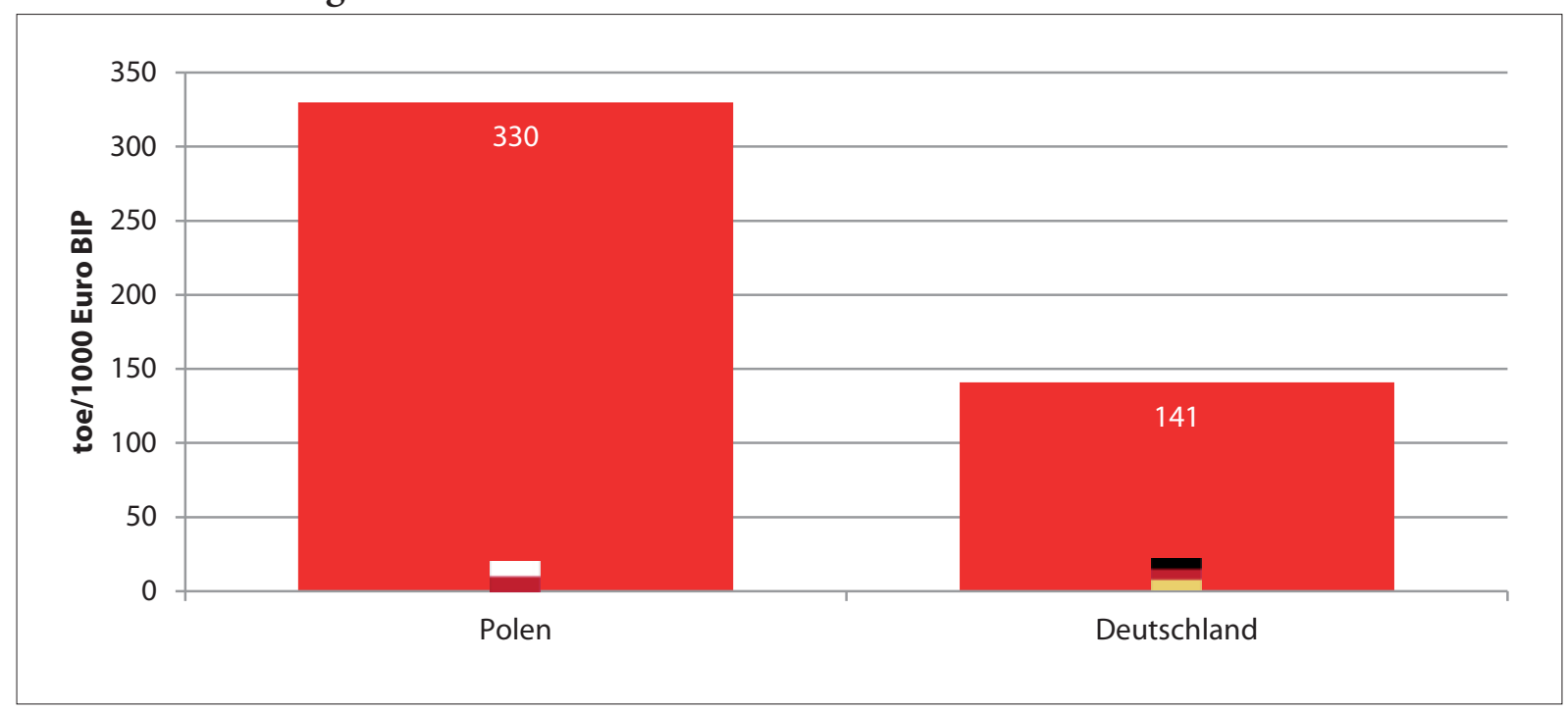

toe - Tonnen Öleinheiten, BIP - Bruttoinlandsprodukt

Quelle: Eurostat Pocketbooks: Energy, transport and environment indicators. European Union 2012. <http://epp.eurostat.ec.europa. eu/cache/ITY_OFFPUB/KS-DK-12-001/EN/KS-DK-12-001-EN.PDF> (abgerufen am 15.05.2014).

\section{CHRONIK}

\section{Mai - 19. Mai 2014}

\begin{tabular}{|c|c|}
\hline 06.05 .2014 & $\begin{array}{l}\text { Jarosław Kaczyński, Parteivorsitzender von Recht und Gerechtigkeit (Prawo i Sprawiedliwość - PiS), ruft die } \\
\text { Regierung dazu auf, einen Politikwechsel für die Region Schlesien durchzuführen, der zu Investitionen führe } \\
\text { und gegen den Zusammenbruch der polnischen Kohleförderung gerichtet sei. Polen müsse den Prozess der Rein- } \\
\text { dustrialisierung beginnen, was in Schlesien besonders leicht sei. Am selben Tag findet in Kattowitz ein Kohle- } \\
\text { gipfel mit Ministerpräsident Donald Tusk, Wirtschaftsminister Janusz Piechociński, Experten, Managern und } \\
\text { Gewerkschaftsvertretern über die Lage der Bergbaubranche statt. }\end{array}$ \\
\hline 07.05 .2014 & $\begin{array}{l}\text { Außenminister Radosław Sikorski trifft sich mit NATO-Generalsekretär Anders Fogh Rasmussen zu Beginn } \\
\text { von dessen zweitägigem Besuch in Polen. Gemeinsam mit Verteidigungsminister Tomasz Siemoniak und dem } \\
\text { Chef des Generalstabs, Mieczysław Gocuł, wird der russisch-ukrainische Konflikt thematisiert. Die realisti- } \\
\text { sche Einschätzung der bestehenden Bedrohung und eine entsprechende Anpassung der Aktivitäten der NATO } \\
\text { seien Pflicht, so Sikorski. Weiter unterstreicht er, dass für das Bündnis eine Rückkehr zur klassischen Vertei- } \\
\text { digungsfähigkeit notwendig sei. Das sei entscheidend für die Glaubwürdigkeit der kollektiven Verteidigung. }\end{array}$ \\
\hline 08.05 .2014 & $\begin{array}{l}\text { Staatspräsident Bronisław Komorowski empfängt NATO-Generalsekretär Anders Fogh Rasmussen. Die ange- } \\
\text { messenste Antwort der NATO auf die russisch-ukrainische Krise sei die Präsenz der NATO an der Ostflanke } \\
\text { des Bündnisses, so Komorowski. Rasmussen kündigt an, dass mit NATO-Übungen, Verteidigungsplänen und } \\
\text { Verlegung von militärischen Einheiten die Sicherheit in den östlichen NATO-Staaten weiter gestärkt werde. }\end{array}$ \\
\hline 09.05 .2014 & $\begin{array}{l}\text { In Warschau beraten Ministerpräsident Donald Tusk und der Präsident der Europäischen Kommission, José } \\
\text { Manuel Barroso, über die Krise zwischen Russland und der Ukraine und das polnische Projekt einer Energie- } \\
\text { union. Dieses wird im Juni auf der Tagesordnung des Europäischen Rates stehen. }\end{array}$ \\
\hline 10.05 .2014 & $\begin{array}{l}\text { Bei einem Treffen in Krakau sprechen Staatspräsident Bronisław Komorowski und der Präsident der Europäi- } \\
\text { schen Kommission, José Manuel Barroso, über die Lage in der Ukraine. Komorowski äußert sich beunruhigt } \\
\text { über die offene Zurschaustellung militärischer Stärke von Seiten Russlands in der russisch-ukrainischen Krise. } \\
\text { Barroso sagt der Ukraine die Unterstützung der EU zu und kündigt eine Verständigung innerhalb der EU über } \\
\text { weitere Sanktionen gegenüber Russland an. }\end{array}$ \\
\hline
\end{tabular}




\begin{tabular}{|c|c|}
\hline 12.05 .2014 & $\begin{array}{l}\text { Ministerpräsident Donald Tusk spricht sich gegen eine Anerkennung der Referenden aus, die am Vortag in den } \\
\text { ostukrainischen Gebieten Donezk und Lugansk über die Abspaltung von der Ukraine durchgeführt wurden. }\end{array}$ \\
\hline 13.05 .2014 & $\begin{array}{l}\text { Der ukrainische Landwirtschaftsminister Ihor Shvaika sagt zu, dass die Ukraine das Embargo für den Import } \\
\text { von polnischem Schweinefleisch aufheben werde, sobald Experten festgestellt hätten, dass keine Gefahr für die } \\
\text { ukrainische Schweinezucht bestünde. Das Embargo wurde von der Ukraine im Februar verhängt, nachdem in } \\
\text { Polen zwei von der sogenannten Afrikanischen Schweinepest infizierte Wildschweine entdeckt worden waren. } \\
\text { Ministerpräsident Donald Tusk forderte die Ukraine im April auf zu entscheiden, ob sie sich an Russland ori- } \\
\text { entieren wolle, das ebenfalls einen Importstopp verhängt hatte, oder ob sie sich für Polen aussprechen und das } \\
\text { Einfuhrverbot aufheben wolle. }\end{array}$ \\
\hline 14.05 .2014 & $\begin{array}{l}\text { Bei einem Treffen mit dem Ministerpräsidenten von Moldawien, Iurie Leancă, in Warschau lobt Ministerpräsi- } \\
\text { dent Donald Tusk die Maßnahmen Moldawiens für die Annäherung an die EU. Beide Regierungschefs unter- } \\
\text { zeichnen einen Kreditvertrag über } 100 \text { Mio. Euro, den Polen Moldawien zur Unterstützung des Unternehmens- } \\
\text { sektors und der Landwirtschaft gewährt. }\end{array}$ \\
\hline 15.05 .2014 & $\begin{array}{l}\text { Ministerpräsident Donald Tusk nimmt in Bratislava an einem Treffen der Regierungschefs der Visegrád-Gruppe } \\
\text { (Polen, Slowakei, Tschechien, Ungarn) teil und warnt davor, dass Europa die Schlacht um seine Zukunft ver- } \\
\text { liere, sollte kein gemeinsamer Standpunkt in den Bereichen Verteidigung und Energie eingenommen werden. } \\
\text { Mit Bezug auf die von ihm vorgeschlagene Energieunion sagt Tusk, dass die EU eine bessere Verhandlungspo- } \\
\text { sition gegenüber Russland habe, wenn sie alternative Energiequellen und Partner habe. Die verstärkte Anwesen- } \\
\text { heit der NATO in Osteuropa verringere das Konfliktrisiko, anstatt es zu erhöhen, zeigte sich Tusk überzeugt. }\end{array}$ \\
\hline 16.05 .2014 & $\begin{array}{l}\text { Nach Verhandlungen mit Gewerkschaftsvertretern der Bergbaubranche teilt Ministerpräsident Donald Tusk mit, } \\
\text { dass dem Sejm ein Gesetzesvorschlag zur Aussetzung von Zahlungen des Kohlekonzerns Kompania Węglowa } \\
\text { (Kattowitz) an die Sozialversicherungsanstalt (Zakład Ubezpieczeń Społecznych - ZUS) vorgelegt wird. Die } \\
\text { Zahlungen reichen in die Jahre der Umstrukturierung der Kohlebranche und der Gründung des Kohlekonzerns } \\
\text { zurück. Es werde versucht, die finanzielle Sicherheit der Kompania Węglowa zu gewährleisten, da diese sowohl } \\
\text { für die soziale Situation in Schlesien als auch für die Energiesicherheit Polens von fundamentaler Bedeutung } \\
\text { sei. Ein nächstes Treffen soll in den kommenden Wochen stattfinden. }\end{array}$ \\
\hline 17.05 .2014 & $\begin{array}{l}\text { Auf dem Parteitag der Demokratischen Linksallianz (Sojusz Lewicy Demokratycznej-SLD) spricht sich SLD- } \\
\text { Chef Leszek Miller für eine Reindustrialisierung Europas, Mindestlöhne in allen EU-Mitgliedsländern und } \\
\text { eine Finanztransaktionssteuer aus. }\end{array}$ \\
\hline 19.05 .2014 & $\begin{array}{l}\text { Nach neuesten Angaben des Statistischen Hauptamtes (Główny Urząd Statystyczny - GUS) betrug der durch- } \\
\text { schnittliche Monatslohn im Unternehmenssektor im April 3.977,8 Zloty (zirka } 948 \text { Euro), was eine Steigerung } \\
\text { um 3,8\% im Vergleich zum Vorjahreszeitraum ist. }\end{array}$ \\
\hline
\end{tabular}


Die Polen-Analysen erscheinen zweimal monatlich als E-Mail-Dienst. Sie werden gemeinsam vom Deutschen PolenInstitut Darmstadt, der Bremer Forschungsstelle Osteuropa und der Deutschen Gesellschaft für Osteuropakunde in Partnerschaft mit dem Willy Brandt Zentrum für Deutschland- und Europastudien an der Universität Wroclaw (Breslau) herausgegeben.

Ein Archiv der Polen-Analysen finden Sie im Internet unter <www.laender-analysen.de/polen>

Kostenloses Abonnement unter <http://www.deutsches-polen-institut.de/Newsletter/subscribe.php>

Diese Analysen finden Sie online als Lizenzausgabe auf $<$ bpb.de>

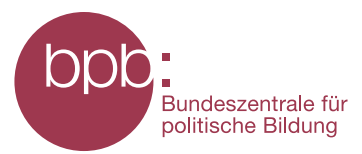

\section{Deutsches Polen-Institut Darmstadt}

Das Deutsche Polen-Institut Darmstadt (DPI) ist ein Forschungs-, Informations-, und Veranstaltungszentrum für polnische Kultur, Geschichte, Politik, Gesellschaft und die deutsch-polnischen Beziehungen, die sich im Kontext der europäischen Integration entwickeln. Das seit März 1980 aktive und bis 1997 von Gründungsdirektor Karl Dedecius geleitete Institut ist eine Gemeinschaftsgründung der Stadt Darmstadt, der Länder Hessen und Rheinland-Pfalz sowie des Bundes. 1987 wurden die Kultusminister der Länder und 2011 das Auswärtige Amt weitere institutionelle Träger. Einen wesentlichen Beitrag zur Verwirklichung der Institutsziele leisten private Stiftungen. Das DPI hat satzungsgemäß die Aufgabe, durch seine Arbeit zur Vertiefung der gegenseitigen Kenntnisse des kulturellen, geistigen und gesellschaftlichen Lebens von Polen und Deutschen beizutragen.

Ziel der Vermittlertätigkeit des DPI ist es, „die zu interessieren, auf die es politisch, wirtschaftlich, gesellschaftlich und kulturell im deutsch-polnischen Verhältnis ankommt« (Leitlinien 1997). Es geht um die Entscheider und Multiplikatoren in Politik, Kultur, Bildung, Verwaltung, Medien und Wirtschaft und, wesentlich stärker ausgeprägt als bisher, um das Hineinwirken in Wissenschaft, Forschung und Bildung.

Derzeit bemüht sich das DPI in Kooperation mit den verstreuten Orten wissenschaftlicher Polen-Kompetenz an deutschen Hochschulen und Forschungsinstituten verstärkt darum, ausgehend von einer Bestandsaufnahme deutscher Polen-Forschung Ort wissenschaftlicher Forschung und verbindendes, vernetzendes und kooperierendes Zentrum zu werden. Ausgangspunkt der Neuausrichtung ist die kaum mehr kontrollierbare Dynamik des Rückbaus der Ressourcen der wissenschaftlichen Polen-Kompetenz in den unterschiedlichen Disziplinen. Mit der über 60.000 Bände zählenden multidisziplinären Fachbibliothek für Polen, die eine einzigartige Sammlung polnischer Literatur in der Originalsprache und in deutscher Übersetzung umfasst, ist das DPI bereits ein geschätzter Ort der Recherche und des wissenschaftlichen Arbeitens. (www.deutsches-polen-institut.de)

Forschungsstelle Osteuropa an der Universität Bremen (www.forschungsstelle.uni-bremen.de)

1982 gegründet, widmet sich die Forschungsstelle Osteuropa an der Universität Bremen der interdisziplinären Analyse der Länder Ost- und Ostmitteleuropas in Zeitgeschichte und Gegenwart. Der Forschungsschwerpunkt liegt dabei auf der Rolle von »Dissens und Konsens«, von Opposition und Zivilgesellschaft in ihrem historischen, politischen, gesellschaftlichen und kulturellen Kontext. Die Forschungsstelle besitzt in ihrem Archiv eine einzigartige Sammlung alternativer Kulturgüter und unabhängiger Texte aus den ehemaligen sozialistischen Ländern. Darunter befindet sich auch eine umfangreiche Sammlung des "Zweiten Umlaufs«, die das Schrifttum und Dokumente unabhängiger Initiativen und gesellschaftlicher Gruppen in Polen aus der Zeit von 1976 bis zum Umbruch umfasst. Hinzu kommt eine umfangreiche Bibliothek mit wissenschaftlicher Literatur. Mit Archiv, Bibliothek und zwei wissenschaftlichen Abteilungen ist die Forschungsstelle auch eine Anlaufstelle sowohl für Gastwissenschaftler als auch für die interessierte Öffentlichkeit.

Eine der Hauptaufgaben der Forschungsstelle ist die Information der interessierten Öffentlichkeit. Dazu gehören unter anderem regelmäßige E-Mail-Informationsdienste für Politik, Wirtschaft, Zivilgesellschaft und Medien.

\section{Herausgegeben mit finanzieller Unterstützung der Stiftung für deutsch-polnische Zusammenarbeit}

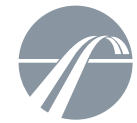

FUNDACJA WSPÓEPRACY

STIFTUNG

STIFTUNG
FUR DEUTSCH-POLNISCHE
ZUSAMMENARBEIT

Die Meinungen, die in den Polen-Analysen geäußert werden, geben ausschließlich die Auffassung der Autoren wieder. Abdruck und sonstige publizistische Nutzung sind nach Rücksprache mit der Redaktion gestattet. Redaktion: Prof. Dr. Dieter Bingen (verantwortlich) (Darmstadt), Silke Plate, M.A. (Bremen) Technische Gestaltung: Matthias Neumann

Polen-Analysen-Layout: Cengiz Kibaroglu, Matthias Neumann

Alle Ausgaben der Polen-Analysen sind mit Themen- und Autorenindex archiviert unter www.laender-analysen.de

Die Polen-Analysen werden im Rahmen der Datenbank World Affairs Online (WAO) ausgewertet und sind im Portal IREON www.ireon-portal.de recherchierbar.

ISSN 1863-9712 @ 2014 by Deutsches Polen-Institut Darmstadt und Forschungsstelle Osteuropa, Bremen

Kontakt: Dr. Andrzej Kaluza, Presse- und Öffentlichkeitsarbeit, Deutsches Polen-Institut, Mathildenhöhweg 2,

D-64287 Darmstadt, Tel.: 06151/4985-13, Fax: 06151/4985-10, E-Mail: polen-analysen@dpi-da.de, Internet: www.laender-analysen.de/polen 